\title{
A Reliable Design of Wireless Body Area Networks
}

\author{
Jocelyne Elias $^{1}$, Abdallah Jarray ${ }^{2}$, Javier Salazar ${ }^{1}$, Ahmed Karmouch ${ }^{2}$, Ahmed Mehaoua ${ }^{1}$ \\ ${ }^{1}$ LIPADE, Université Paris Descartes-Sorbonne Paris Cité, 45 rue des Saints Pères, Paris, France \\ ${ }^{2}$ SITE, University of Ottawa, 800 King Edward, Ottawa, Ontario, K1N 6N5 Canada \\ Email:jocelyne.elias, ahmed.mehaoua@ parisdescartes.fr, ajarray@uottawa.ca, Karmouch@site.uottawa.ca
}

\begin{abstract}
In this paper, we propose a reliable topology design and provisioning approach for Wireless Body Area Networks (named RTDP-WBAN) that takes into account the mobility of the patient while guaranteeing a reliable data delivery required to support healthcare applications' needs. To do so, we first propose a 3D coordinate system able to calculate the coordinates of relay-sensor nodes in different body postures and movements. This system uses a 3D-model of a standard human body and a specific set of node positions with stable communication links, forming a virtual backbone. Next, we investigate the optimal relay nodes positioning jointly with the reliable and cost-effective data routing for different body postures and movements. Therefore, we use an Integer Linear Programming (ILP) model, that is able to find the optimal number and locations of relay nodes and calculate the optimal data routing from sensors and relays towards the sink, minimizing both the network setup cost and the energy consumption. We solve the model in dynamic WBAN (Stand, Sit and Walk) scenarios, and compare its performance to other relaying approaches. Experiment results showed that our realistic and dynamic WBAN design approach significantly improves results obtained in the literature, in terms of reliability, energy-consumption and number of relays deployed on the body.
\end{abstract}

Keywords-WBAN, Topology design, Positioning, 3D-model, Optimization, Reliability, Energy-efficiency.

\section{INTRODUCTION}

Recent advance in wireless technology, networking and embedded sensors has led to the development of Wireless Body Area Network (WBAN) as an emerging technology to improve the quality of human life [1]-[2]. In healthcare area, a WBAN is setup through a number of miniaturized sensors on, in or near the human body. These sensors collect and report data via a wireless communication channel towards a remote processing and monitoring device called sink. Accordingly, the use of a WBAN will enable continuous and remote monitoring of patient's physiological signals, and this could be benefic to trigger first aid assistance and to detect emergency situations [3]-[4].

Since WBANs are deployed in compact region along the human body, and hence the distance between sensors and the sink is relatively short, in literature, many proposals [5]-[6] have assumed a star topology and single hop communication mode for WBANs. However, in [7]-[8], it was concluded that in a WBAN, a multi-hop communication is most reliable and sometimes an utter requirement. Effectively, to reach the sink from a further away sensor node in a star topology, one solution could be to use a channel with higher transmission power. However, a high energy wave may heat and damage body tissue by energy absorption, accordingly multi-hop channels are required to guarantee both low transmission power and reliable data routing. Similarly, authors in [10] concluded that multi-hop communication turns out the most energy-efficient since using a relaying structure improves energy consumption largely, by distributing the routing load over the entire network. Therefore, the idea to add relay nodes in WBAN comes in to use. These special devices will play the role of intermediate nodes that send through multi-hop paths all sensory data towards the sink, thus improving the WBAN lifetime.

Evidently, the deployment of WBAN remains the most important issue that impacts the network lifetime. In general, sensor nodes have pre-fixed locations. Hence, a realistic WBAN topology design needs to optimize the number and the location of relay nodes along with an optimal cost-effective and reliable routing of medical/vital signs data through the network. However, it is not a trivial task in case of the wireless environment on a moving human body. Authors in [10] observed that body limbs have different radio propagation and link characteristics, therefore an energy-efficient routing algorithm must give a particular concern to the localization of relay nodes.

In literature few works have considered the WBAN topology design problem [11]-[13]. The authors of those references looked at the network design from routing and energyefficiency perspective. Very often, it is assumed that the number of relay nodes is pre-determined, and their strategic physical locations are not optimized. Hence, they do not minimize the overall network cost, and they often deploy much more networking resources than needed. That means additional cost and complexity.

To address these concerns, we propose in this work a reliable topology design and provisioning approach for WBAN, called RTDP-WBAN, that takes into account the mobility of the patient while guaranteeing a reliable data delivery required to support healthcare applications' needs. We consider a multi-hop WBAN topology, combined with a multi-hop communication, wherein a set of relay nodes is distributed along the body to serve as transport network for sensory and control data. We call this relaying structure virtual backbone network where each node has some fixed neighbors to which it has constant distance and has relatively stable and reliable links in the presence of postural mobility related to body movement [12], [13].

RTDP-WBAN approach uses a 3D In-Body Positioning System (3D-IBPS) inspired by the virtual coordinate space proposed in [13]. Within this IBPS, each node position along the human body is identified through a triplet of coordinates. Owing to investigate the impact of body movement on WBAN topology design as well as on energy consumption, we propose a set of candidate regions for the positioning of relay nodes based on: (i) the specific radio propagation of each body limb, 
(ii) the range constraints of each body physical movement, i.e., sit, stand and walk, and (iii) the required vital signal measurements. These regions will be related by a backbone structure which is relatively stable and reliable with respect to body movement. To determine the optimal low-cost and energy-efficient WBAN topology, RTDP-WBAN uses an ILP model developed in our previous work [14], that is able to: (i) find the optimal number and locations of relay nodes, (ii) calculate the optimal data routing from sensors and relays towards sink, and (iii) minimize the network setup cost and the sensing/relaying energy consumption.

The remainder of the paper is organized as follows. Section II presents related works. Section III describes the proposed reliable and dynamic low-cost and energy-efficient WBAN design approach. Section IV lists the proposed performance evaluation metrics, followed by the numerical results. Finally, Section V concludes the paper.

\section{RELATED WORK}

In literature, several proposals for an efficient design of wireless sensor and ad hoc networks have been proposed in several contexts [5]-[9]. However, these approaches are unadapted for WBANs and need to be significantly improved. Few works have considered the WBAN topology design problem with the purpose of providing a low-cost and energyefficient resource management approach.

Authors in [10] proposed two mechanisms based on relaying node communication architecture to improve the network lifetime. A first solution, called relaying, is presented, where a dedicated relaying structure is used to forward data towards the sink. The second solution, called cooperation, is proposed as a trade-off between single and multi-hop communication modes. However, relaying nodes as well as their positioning in both mechanisms are fixed and not optimized.

The approach proposed in [11] is also based on relaying to improve WBAN lifetime. Authors proposed a heuristic approach to upper bound the number of relaying nodes based on path loss coefficients of human body. However, as authors do not aim to minimize the overall network cost, positioning of relaying nodes is pre-determined.

Relaying has been also considered in [12], where WBAN is studied in the context of body mobility. To guarantee a reliable data routing, they proposed a relaying structure which has relatively stable links in presence of body movement. However, the number of relays as well as their positioning is pre-determined. Accordingly, the network cost is not minimized and most often much more resources than required are deployed.

Similarly, more recently Zhang et al. [13] proposed a positioning system able to calculate the coordinates of relay nodes within a virtual coordinate body space in different body postures and movements. However, as the focus of this work is on the development of a reliable routing protocol to increase the network lifetime, the number of relay nodes is pre-determined and the relaying cost is not optimized.

To address these concerns, we have proposed in our previous work [14] an optimization framework that minimizes relaying cost and maximizes energy efficiency while considering connectivity constraints in WBAN for the scenario of body standing posture. In this work, we extend the previous one taking into account the effect of different postures resulting from body movement on the WBAN topology design in terms of relay node positioning, cost and energy consumption. We aim to come-up with a realistic and dynamic WBAN topology design approach that can guarantee a consistent performance regarding human body movement.

\section{RELIABLE TOPOLOGY DESIGN AND PROVISIONING (RTDP-WBAN) APPROACH}

\section{A. Reliable WBAN topology}

1) Proposed WBAN architecture: As aforementioned dedicated relaying architecture turns out the most energy-efficient and most reliable. In this architecture, we identify three types of nodes: a sink node, a set of sensor nodes, and a set of relay nodes distributed along the body to serve as a transport network for sensory and control data.

a) Sensor positioning: Sensor nodes are placed at fixed location along the patient's body in order to capture Heart condition, Temperature, Blood pressure, Pulse, Glucose and Motion (as illustrated in Figure 2c).

b) Potential relays' positioning zones: To setup a WBAN topology using a multi-hop communication architecture, we identify, as in [12], 8 regions which are illustrated in Figure 1a. These regions are proposed to handle relay nodes as it is expected that links between these regions will provide relatively stable and reliable communication in the presence of postural mobility related to body physical movement. Indeed, the identification of these potential relay locations is based on contextual knowledge obtained from dynamic patient body position and motion that results in changing of inter-node positions and wireless channel communication.

c) Virtual transport backbone: To guarantee a reliable communication between sensors, relays and sink node, we will identify for each body posture or movement an optimal virtual transport backbone topology, based on the identified candidate relays' positions. Figure $1 \mathrm{~b}$ shows the proposed backbone relaying topology, in which each node has some fixed neighbors to which it has constant Euclidean distance. A communication link between two identified neighbors is called backbone link. We note that these backbone links connect all the network nodes together. In addition, this backbone structure has relatively stable and reliable links in the presence of postural mobility and/or the range constraints of each body physical movement. Doing so, desired reliability and resource management can be achieved according to static or dynamic body positions. By correlating body movement and adaptive data routing, topological choice in terms of number and relay nodes positioning will be made according to the spatial-temporal association among connected WBAN nodes.

2) $3 D$ In-Body Positioning System (3D-IBPS): The $3 D$ system is used to model body movement and postures. We use an $X Y Z$ coordinate system and we assume that the origin (the point $(0,0,0))$ is located in the sink $S$, as shown in Figure 1c. Each node position within the human body is identified through the triplet $(x, y, z) . x$ and $y$ are the coordinates in the plan. $z$ coordinate refers to the third dimension and it is used to: (i) include contextual knowledge obtained from dynamic 
patient body position, and (ii) capture the changes in inter-node positions and wireless communication channel related to body movement, such as sit and walk. We show in Figures $2 \mathrm{a}$ and $2 \mathrm{~b}$ the application of 3D-IBPS to calculate the coordinates for candidate relaying zones in case of stand posture. The distance $d$ between two nodes $A=\left(x_{A}, y_{A}, z_{A}\right)$ and $B=\left(x_{B}, y_{B}, z_{B}\right)$, as shown in Figure $1 \mathrm{c}$, is calculated using the Euclidean formula: $d=\sqrt{\left(x_{B}-x_{A}\right)^{2}+\left(y_{B}-y_{A}\right)^{2}+\left(z_{B}-z_{A}\right)^{2}}$.

\section{B. Low-cost and energy-efficient WBAN provisioning ap- proach}

We define in this section our proposed WBAN provisioning model that will choose the topology that optimizes the cost and required sensing/relaying energy.

1) Network model: A common approach to the network design problem is to consider feasible positions where relays can be installed (Candidate Sites, CSs) [15]. Sensors and sink positions (e.g., arms, legs, breast, ...) are usually predetermined and fixed, according to the medical application for which they are deployed.

Let $S$ and $P$ denote the set of sensors and CSs, respectively, and $o$ the sink node index.

Each relay can establish a wireless link with any other relay located within its communication range, $R_{c}$, as well as with any sensor at a distance lower than $R_{s}$ (the sensor radio range), where $R_{c} \geq R_{s}$, in general. For each sensor $i$, we define the ordered vector $O R_{i}$ of the reachable wireless relays. These relays are ordered from the closest to the farthest with respect to sensor $i$. The $j$-th and $k$-th element of $O R_{i}$ are given by $O R_{i}(j)$ and $O R_{i}(k)$, respectively, and they indicate the relays at the $j$-th and $k$-th place in the vector. So, if $j<k$, then $O R_{i}(j)$ is closer to sensor $i$ than relay $O R_{i}(k)$. Let us denote by $I_{i}$ the index set of the ordered vector $O R_{i}$. The cost related to the setup of a relay in $\operatorname{CS} j$ is denoted by $c_{j}^{I}$, and its capacity is denoted by $v_{j}, \forall j \in P$. Furthermore, the traffic generated by sensor $i$ towards the sink $o$ is given by $d_{i o}, i \in S$.

According to sensors, sink and CSs location, the following connectivity parameters can be calculated. Let $a_{i j}, i \in S$, $j \in P$, and $e_{j o}, j \in P$ denote the sensor and the sink coverage parameters, respectively. $a_{i j}=1$ indicates that sensor $i$ can establish a link with a relay installed in CS $j$, and 0 otherwise. Similarly, the parameter $e_{j o}=1$ indicates that a relay installed in CS $j$ can establish a link with the sink $o$, and 0 otherwise. Obviously, $a_{i j}$ depends on the proximity of sensor $i$ to $\mathrm{CS} j$, as well as on the propagation conditions between such nodes, and $e_{j o}$ is related to the distance between CS $j$ and sink $o$.

Finally, let $b_{j l}, j, l \in P$ denote the connectivity parameters between two different CSs, which may depend on the proximity of the relays $j$ and $l$ in the network; $b_{j l}=1$ states that CS $j$ and $l$ can be connected with a wireless link, and 0 otherwise.

Decision variables of the problem include sensor assignment variables $x_{i j}, i \in S, j \in P$, and relays' installation variables $z_{j}, j \in P$. Binary variable $x_{i j}=1$ indicates that sensor $i$ is assigned to $\mathrm{CS} j$, and binary variable $z_{j}=1$ indicates that a relay is installed in CS $j$. Finally, flow variables $f_{j l}^{o}$ which denote the traffic flow routed on link $(j, l)$ destined to the sink $o$. The special variables $f_{j o}^{t}$ denote the total traffic flow between the relay installed in CS $j$ and the sink $o$.
To calculate the energy consumption in wireless nodes, we adopt the propagation model used in [10], [11]. Therefore, the sensing and processing energy are negligible with respect to communication energy, and hence, the total energy consumption is represented by the total transmission and reception energy of all wireless nodes. The energy the radio dissipates to run the circuitry for the transmitter and receiver are denoted by $E_{T X \text { Xec }}$ and $E_{R X e l e c}$, respectively. The path loss coefficient on the wireless link between nodes $i$ and $j$ is denoted by $n_{i j}$. $E_{a m p}\left(n_{i j}\right)$ is the energy for the transmit amplifier, and $D_{i j}$ is the distance between nodes $i$ and $j$. The transmission energy can therefore be computed as: $w\left[E_{T X \text { elec }}+E_{a m p}\left(n_{i j}\right) D_{i j}^{n_{i j}}\right]$ while the reception energy is $w E_{R X e l e c}$, where $w$ is the total number of transmitted/received bits.

2) RTDP-WBAN ILP model: Given the above notations, parameters and variables, we now illustrate our proposed reliable topology design and provisioning WBAN model (RTDPWBAN), which minimizes at the same time the total network installation cost and the overall energy consumed by the network, while ensuring full coverage of all sensors and effective routing of medical data towards sink node. The RTDP-WBAN ILP model is defined as follows:

$$
\begin{aligned}
\min & \left\{\sum_{j \in P} c_{j}^{I} z_{j}+\alpha\left(\sum_{i \in S, j \in P} d_{i o} x_{i j}\left(E_{T X e l e c}+E_{a m p}\left(n_{i j}\right) D_{i j}^{n_{i j}}\right)+\right.\right. \\
& +\sum_{i \in S, j \in P} d_{i o} x_{i j} E_{R \text { Xelec }}+ \\
& +\sum_{j, l \in P} f_{j l}^{o}\left(E_{T X \text { Xelec }}+E_{a m p}\left(n_{j l}\right) D_{j l}^{n_{j l}}+E_{R X e l e c}\right)+ \\
& \left.\left.+\sum_{j \in P} f_{j o}^{t}\left(E_{T X e l e c}+E_{a m p}\left(n_{j o}\right) D_{j o}^{n_{j o}}+E_{R X e l e c}\right)\right)\right\}
\end{aligned}
$$

s.t.

$$
\begin{array}{lr}
\sum_{j \in P} x_{i j}=1, & \forall i \in S \text { (2) } \\
x_{i j} \leq z_{j} a_{i j}, & \forall i \in S, j \in P \text { (3) } \\
\sum_{i \in S} d_{i o} x_{i j}+\sum_{l \in P}\left(f_{l j}^{o}-f_{j l}^{o}\right)-f_{j o}^{t}=0, & \forall j \in P \text { (4) } \\
f_{j l}^{o} \leq \sum_{i \in S} d_{i o} b_{j l} z_{j}, f_{j l}^{o} \leq \sum_{i \in S} d_{i o} b_{j l} z_{l}, & \forall j, l \in P_{(5)} \\
\sum_{i \in S} d_{i o} x_{i j}+\sum_{l \in P} f_{l j}^{o} \leq v_{j}, & \forall j \in P \text { (6) } \\
f_{j o}^{t} \leq \sum_{i \in S} d_{i o} e_{j o} z_{j}, & \forall j \in P \text { (7) } \\
z_{O R_{i}(a)}+\sum_{b \in I_{i}: b>a} x_{i O R_{i}(b)} \leq 1, & \forall i \in S, a \in I_{i} \text { (8) } \\
x_{i j}, z_{j} \in\{0,1\}, & \forall i \in S, j \in P^{(9)}
\end{array}
$$

The objective function (1) accounts for the total setup cost and the total energy consumption. The first term, $\sum_{j \in P} c_{j}^{I} z_{j}$, takes into account the relay nodes installation cost, while the second term represents the total energy consumed by the network (relays and sensors), including the transmission and reception energy, $\alpha$ being a parameter that permits to give more weight to one component with respect to the other. For big $\alpha$ values, the first component becomes negligible and the model minimizes only the energy consumed by the network. On the other hand, for small $\alpha$ values the model minimizes the relays' installation costs. 
More in detail, the second term of objective function (1) is composed of the following elements: $\sum_{i \in S, j \in P} d_{i o} x_{i j}\left(E_{T X \text { Xec }}+E_{a m p}\left(n_{i j}\right) D_{i j}^{n_{i j}}\right)$ as the total energy consumed by all sensors to transmit data to relays, and $\sum_{i \in S, j \in P} d_{i o} x_{i j} E_{R X \text { elec }}$ which is the total energy consumed by relays to receive data from all sensors. The terms $\sum_{j, l \in P} f_{j l}^{o}\left(E_{T X \text { Xec }}+E_{a m p}\left(n_{j l}\right) D_{j l}^{n_{j l}}\right)$ and $\sum_{j \in P} f_{j o}^{t}\left(E_{T X \text { Xec }}+E_{a m p}\left(n_{j o}\right) D_{j o}^{n_{j o}}\right)$ are the total energy consumed by relays to forward data to other relays and to sink, respectively. Finally $\sum_{j, l \in P} f_{j l}^{o} E_{R X e l e c}$ is the total energy that relays dissipate for receiving data from other relays, while $\sum_{j \in P} f_{j o}^{t} E_{R X \text { elec }}$ is the total energy consumed by sink node $o$ to receive the corresponding data collected by all sensors from relays.

Constraints (2) provide full coverage of all sensors, while constraints (3) are coherence constraints ensuring that a sensor $i$ can be covered by CS $j$ only if a relay is installed in $j$ and if $i$ can be connected to $j$. Constraints (4) define the flow balance in relay node $j$ for all the traffic destined towards sink node $o$. These constraints are similar to those adopted for classical multi-commodity flow problems: the term $\sum_{i \in S} d_{i o} x_{i j}$ is the total traffic generated by the covered sensors destined towards sink node $o, \sum_{l \in P} f_{l j}^{k}$ is the total traffic received by relay $j$ from neighboring nodes, $\sum_{l \in P} f_{j l}^{o}$ is the total traffic transmitted by $j$ to neighboring nodes, and $f_{j o}^{t}$ is the traffic transmitted towards the sink node $o$. We note that these constraints define the multi-hop paths (i.e., the routing) for all the traffic that is transmitted in the WBAN. Constraints (5) define the existence of a link between CS $j$ and CS $l$, depending on the installation of relays in $j$ and $l$ and the connectivity parameters $b_{j l}$. Constraints (6) impose, for each relay node $j$, that the ingress traffic (from all covered sensors and neighbors) serviced by such network device does not exceed its capacity $v_{j}$, whilst constraints (7) force the flow between relay $j$ and sink $o$ to zero if node $j$ is not connected to $o$. Constraints (8) force each sensor to be assigned to the closest installed relay, and constraints (9) are the integrality constraints for the binary decision variables.

We note that the above model is NP-hard as it includes the set covering and the multi-commodity flow problems as special cases. However, we will demonstrate in the next section that it can be solved to the optimum in a very short computing time (only fractions of a second), thus representing a very effective tool to plan both energy-efficient and cheap wireless body area networks.

\section{NUMERICAL RESULTS}

\section{A. Simulation scenarios}

We simulated the body movement in stand, sit and walk scenarios. We show in the following the effect of body postures and movement on the WBAN topology.

\section{1) Stand and Sit scenarios:}

a) Candidate virtual transport backbone: Stand and sit body postures do not imply an important impact on the virtual topology expected to connect selected relay nodes within the candidate zones. We will simulate these postures using the $3 \mathrm{D}$ IBPS coordinates of candidate relay zones shown in Figures $2 \mathrm{a}$ and $3 \mathrm{a}$, respectively. b) Design algorithm: As mentioned previously, our main goal is to determine the optimal virtual backbone topology that minimizes both the WBAN installation cost and sensory/relaying energy consumption. To do so, we generate a number of random relay positioning configurations within the candidate zones identified previously (see Figure 1a). Next, we call the RTDP-WBAN model to calculate the optimal virtual backbone for each configuration.

2) Walk scenario:

a) Candidate virtual transport backbone: To simulate the walk movement for the human body, we use the different position frames of the sensors on the body, obtained with the software make human [17] on different moments of the movement simulation. To do so, we decompose the walk movement into a set of cycles, where each movement cycle starts from a first and ends at a last frame position. In our experimental setup we will use the three frames depicted in Figure 3b, where we show the 3D-IBPS coordinates of candidate regions to handle the nodes of the relaying virtual backbone. We note that there can be more frames between the first and the last one in order to make the simulation more accurate.

b) Design algorithm: To calculate the optimal low-cost and energy-efficient virtual backbone topology, we proceed as follows. We call the RTDP-WBAN model to calculate the optimal virtual backbone topology for the first frame. Next, using the 3D-IBPS coordinates of the relay locations already defined in frame one and the 3D-IBPS coordinates of sensors in frame 2, we calculate the vector that represents the transition between frame 1 and 2 . Then, we calculate the 3D-IBPS coordinates of candidate regions to handle the relays of frame 2 and we call the RDTP-WBAN ILP model to calculate the optimal virtual backbone topology. Next, we do the same steps for frame 3 .

\section{B. Simulation setup}

In this section, we set up the simulation environment used to evaluate the performance of the proposed 3D-IBPS system and low-cost and energy-efficient WBAN provisioning model.

We limit WBAN devices' transmission range (and as a consequence, the power) assuming that each sensor and the sink can be connected to a candidate relay only if the relay is at a distance not greater than $30 \mathrm{~cm}$ from the sensor or the sink (i.e., $R_{s}=30 \mathrm{~cm}$ ). This transmission range/power limitation is required in WBAN to protect human tissue [3], [16]. We further assume that a CS $j$ can be connected with a wireless link to a CS $l\left(b_{j l}=1\right)$, if either $j$ and $l$ are in the same ellipsoidal area (see Figure 1a) or $l$ is at a distance not greater than $80 \mathrm{~cm}$ from $j$ (i.e., $R_{c}=80 \mathrm{~cm}$ ). We set the installation cost of a relay equal to 10 monetary units, and its maximum capacity equal to $250 \mathrm{~kb} / \mathrm{s}$.

In order to compute the energy consumption, we adopt in this paper the Nordic nRF2401 transceiver which is commonly used in body sensor networks [10], [11]. Hence, the radio dissipates $E_{\text {TXelec }}=16.7$ and $E_{\text {RXelec }}=36.1 \mathrm{~nJ} / \mathrm{bit}$ to run the transmitter and receiver circuitry, respectively. The path loss coefficient $\left(n_{i j}\right)$ takes the value 3.38 (line of sight case) or 5.9 (non line of sight case). The energy for the transmit amplifier $E_{a m p}\left(n_{i j}\right)$ depends on $n_{i j}$, and it is equal to $1.97 \mathrm{~nJ} / \mathrm{bit}$ for $n_{i j}=3.38$ and $7.99 \mu \mathrm{J} / \mathrm{bit}$ for $n_{i j}=5.9$. 


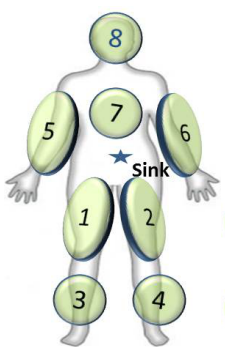

(a)

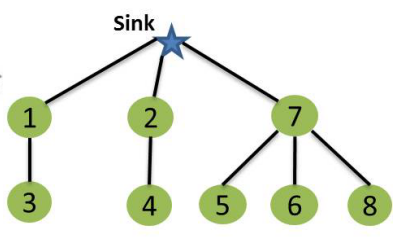

(b)

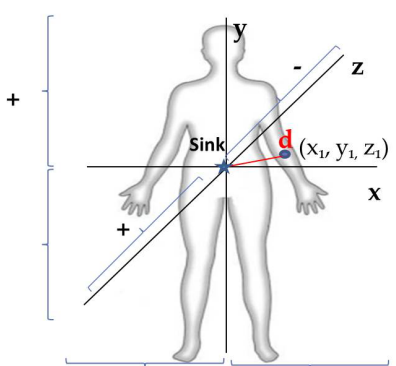

(c)

Figure 1: (a) Candidate zones for placing relays, (b) Virtual backbone network and (c) 3D-IBPS system

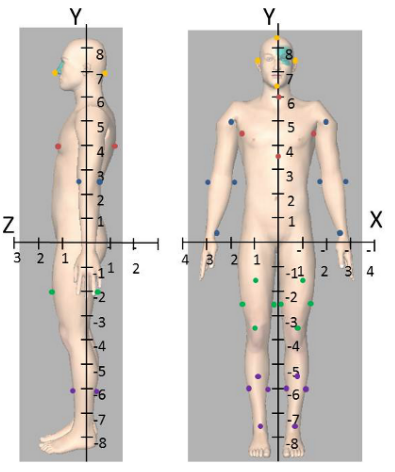

(a)

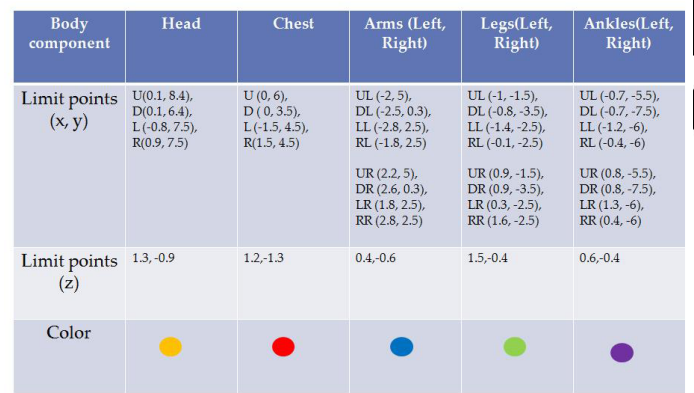

(b)

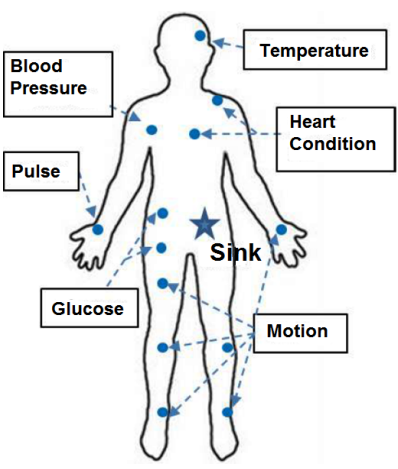

(c)

Figure 2: (a) Decomposition of stand posture, (b) Limits of candidate relays' zones and (c) Sensors positioning

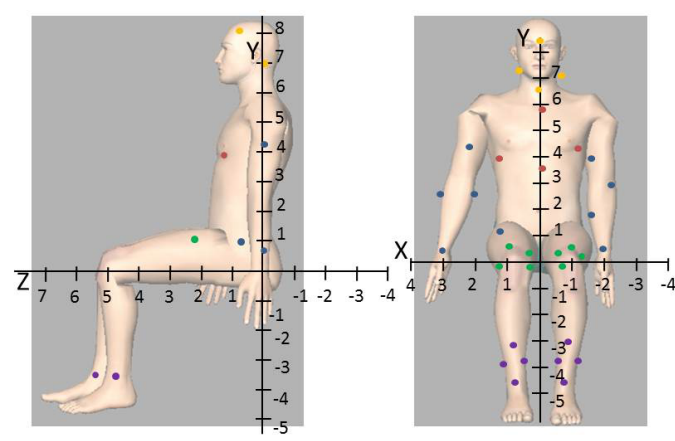

(a) Decomposition of sit posture

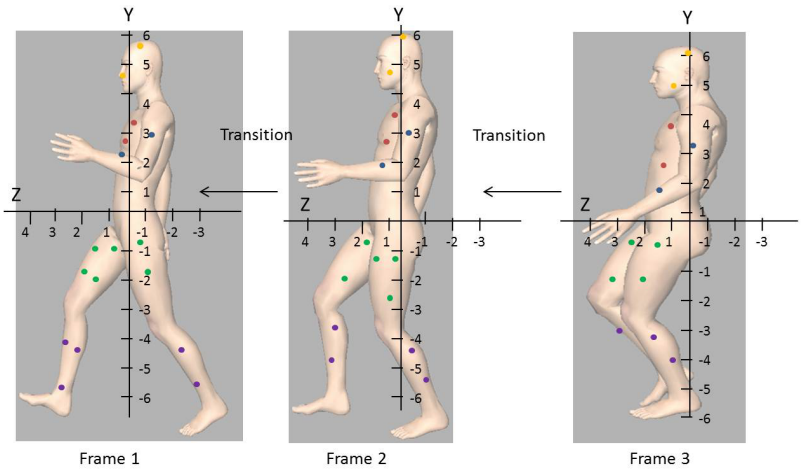

(b) Decomposition of walk posture

Figure 3: WBAN topology in case of sit and walk postures

For each scenario (Stand, Sit and Walk), we generate 100 relaying topology configurations, where each configuration corresponds to a distinct relaying node positioning. To do so, we generate for each relay node random $(x, y, z)$ coordinates within the candidate regions depicted in Figure 1a. We evaluate the performance of the RTDP-WBAN model considering the WBAN topology that consists of 13 sensors (see Figure 2c) and a relaying backbone topology that can use a maximum of 20 candidate relaying nodes. We note that simulations were made using the C++ and CPLEX solver. To achieve a realistic simulation of the human body we used the software Makehuman [17]. This software simulates different human bodies and allows to create different scenarios for body positions and movement.

\section{Used Benchmarks}

To evaluate the performance of our proposed WBAN design approach, we use the two following benchmarks:

- Relay Network: in [11], authors propose an approach called Relay Network in order to forward data towards the sink. A relay network is defined as a network of relays distributed along the body, in conjunction with sensor nodes, to serve as a transport network for the WBAN. Each sensor is connected to this transport network through one short hop. Relaying nodes are added until all sensor and relay nodes have at least one relay node in line of sight.

- Relaying-2 and Relaying-3: in [10], authors propose 


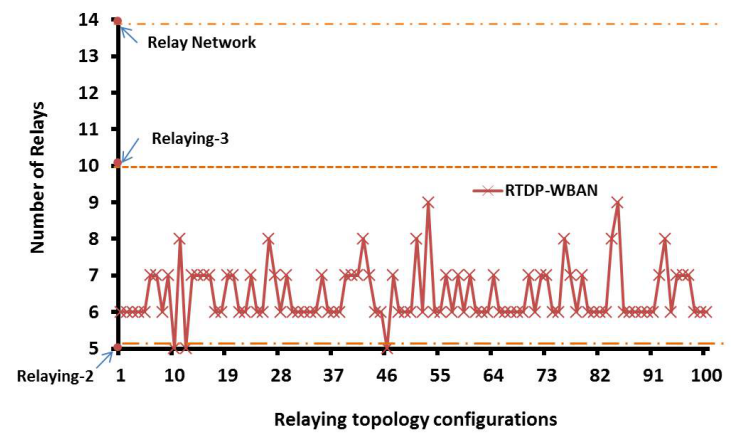

(a) Number of relays

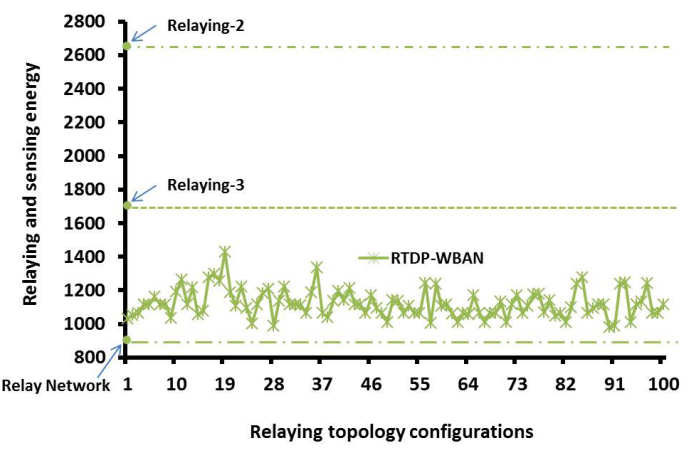

(b) Total energy consumption

Figure 4: Stand Scenario

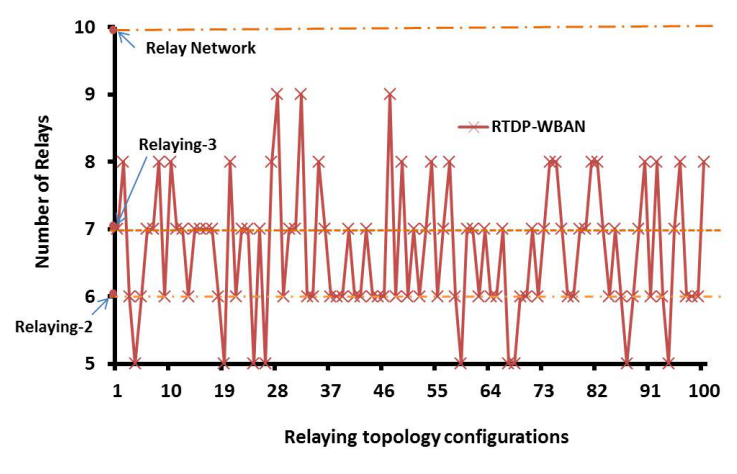

(a) Number of relays

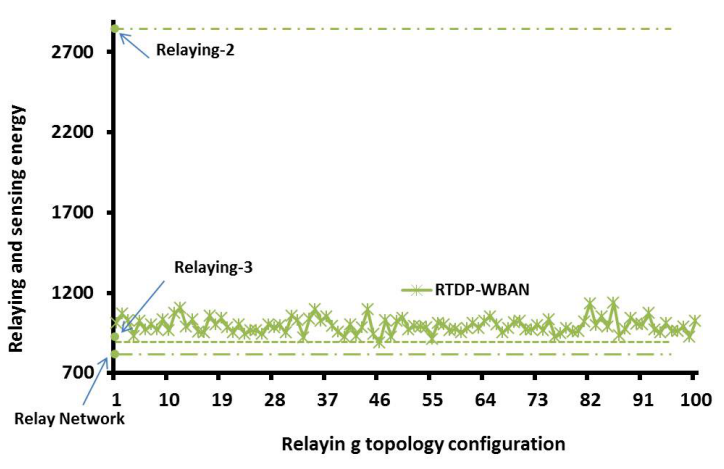

(b) Total energy consumption

Figure 5: Sit Scenario

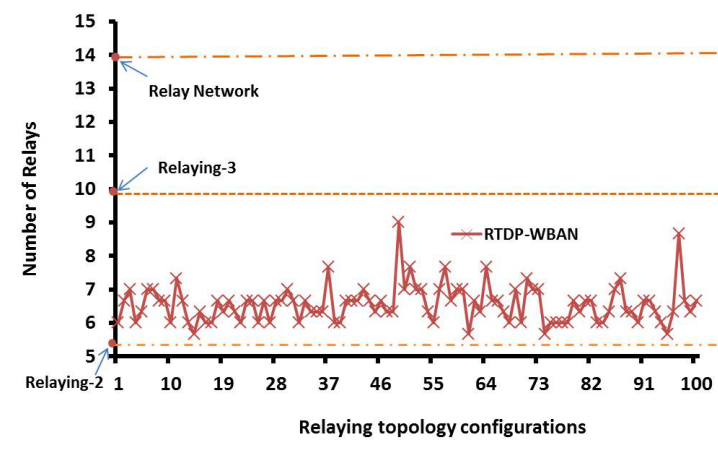

(a) Number of relays

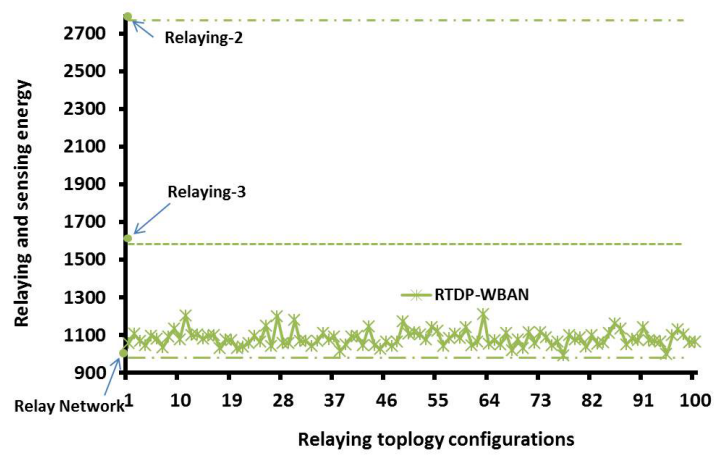

(b) Total energy consumption

Figure 6: Walk Scenario

a dedicated tree-based relaying topology combined with cooperative forwarding in order to improve the network lifetime. Nodes cooperate in forwarding data towards the sink and relaying devices are placed next to exiting nodes, so the inter-node distance remains the same. Proposed approaches called Relaying- 2 and Relaying-3 use a tree-based relaying topology, where relays are placed exclusively at level 2 and level 3, respectively, and they forward the data of the sensors further away from the sink.

\section{Performance evaluation metrics}

To evaluate the performance of our RDTP-WBAN approach, we measure the following metrics:

- Per sensor energy consumption $\left(E_{s}\right)$ : this metric represents the average total energy per bit consumed by each sensor.

- Total energy consumption $\left(E_{t o t}\right)$ : this metric represents the total energy per bit consumed by the network, and is measured as the sum of energies consumed by all sensors and relays. 
- Number of relaying nodes $\left(N_{R}\right)$ : it is the number of relays in the virtual transport backbone structure.

\section{E. Evaluation results}

We now evaluate and compare the RDTP-WBAN model performance to that of Relaying-2 and Relaying-3 [10], as well as Relay Network [11] approach. This comparison is done in the Stand, Sit and Walk scenarios, for 100 different relaying topology configurations, in terms of the already defined performance metrics; $E_{s}, E_{t o t}$ and $N_{R}$.

Figures 4, 5 and 6 show the total number of relays and the total energy consumed by relaying and sensing nodes for 100 different relaying topology configurations, in the case of the Stand, Sit and Walk scenario, respectively. For the Walk scenario, we have decomposed the walking movement into three frames as illustrated in Figure 3b. For each configuration of relaying topology, we have calculated the results of Figure 6 by averaging over the results obtained for the three frames.

For the Stand and Walk scenarios, the results are quite similar; it can be observed that the RTDP-WBAN model exhibits better performance than the Relay Network and Relaying-3 approaches in terms of number of relays, and than the Relaying- 2 and Relaying-3 approaches in terms of energy consumption, for all 100 configurations. Furthermore, it can be seen that the number of relays deployed by our model (on average, $N_{R} \approx 6$ ) is slightly higher than the one obtained by the Relaying- 2 approach $\left(N_{R}=5\right)$, while this latter consumes a lot of energy with respect to the proposed model. Relaying-2 has an energy $E_{t o t} \approx 2622 \mathrm{~nJ} / \mathrm{bit}$ for the Stand scenario and $\approx 2791 \mathrm{~nJ} / \mathrm{bit}$ for the Walk scenario, while our model consumes 981 and $996 \mathrm{~nJ} / \mathrm{bit}$ for the Stand and Walk scenario, respectively. This is due to the fact that the proposed model has been developed in such a way that it succeeds in minimizing both the total energy consumed by the WBAN and the total energy consumed by each sensor, while maintaining the number of relays quite small. Furthermore, a distinguishing feature of our model with respect to [10], [11] is that it reconfigures dynamically the backbone relaying network taking into account the person's movements and body postures, like sitting and walking. The results illustrated in Figure 5 for the Sit scenario show us that the proposed model outperforms the Relay Network and Relaying-2 approach, in terms of number of relays and energy consumption, respectively. While it exhibits the same performance than other approaches in all other cases.

Finally, to summarize, a key feature of the RDTP-WBAN model with respect to all other approaches is that it optimizes the relays' locations not only in realistic candidate regions (as illustrated in Figure 1a), but also dynamically taking into consideration the person's mobility. This is done, while minimizing both the number of relays to be deployed and the total energy consumption. The numerical results prove that our model provides us with a very good tradeoff between the number of relays and the energy consumption.

\section{CONCLusion}

In this paper, we addressed the reliable topology design problem for WBANs, taking into account the movement and different body postures. To this end, we proposed a reliableand-dynamic topology design and energy-efficient resource provisioning approach (RTDP-WBAN), focusing on three specific scenarios (Stand, Sit and Walk). The RTDP-WBAN approach is based on a 3D coordinate system (3D-IBPS), which is able to calculate the $(x, y, z)$ coordinates of sensors and relays in different body postures and movements. We evaluated the performance of the RTDP-WBAN approach, comparing it to two other relaying approaches, in the Stand, Sit and Walk scenarios. The performance metrics adopted in this work were the total energy per bit consumed by the WBAN and by each sensor, and the number of relays deployed on the body. Numerical results showed that the proposed approach is reliable and energy-efficient, and it offers an interesting and flexible tradeoff between the number of relays deployed in the WBAN and the total energy consumption.

\section{REFERENCES}

[1] S. Ullah, H. Higgins, B. Braem, B. Latre, C. Blondia, et al., A comprehensive survey of wireless body area networks, Journal of Medical Systems, 36(3):1065-1094, 2012.

[2] B. Latre, B. Braem, I. Moerman, C. Blondia, and P. Demeester, A survey on wireless body area networks, Journal of Wireless Networks, 17(1):1$18,2011$.

[3] H. Alemdar and C. Ersoy, Wireless sensor networks for health care: A survey, Computer Networks, 54(15):2688-2710, 2010.

[4] P. Neves, M. Stachyra, and J. Rodrigues, Application of wireless sensor networks to health care promotion, Journal of Communications Software and Systems, 4(3):181-190, 2008.

[5] C. Otto, A. Milencovic, C. Sanders, E. Jovanov, System Architecture of a wireless Body Area Sensor Network for Ubiquitous Health Monitoring, Mobile Multimedia, 1(4):307-326, 2006.

[6] G. Virone, et al., An Advanced Wireless Sensor Network for Health Monitoring, in D2H2, 2006.

[7] A. Natarajan, M. Motani, B. De Silva, K.K. Yap and K.C. Chua, Investigating network architectures for body sensor networks, in Healthnet, pp. 19-24, 2007.

[8] A. Natarajan, M. Motani, B. De Silva, K.K. Yap and K.C. Chua, Link layer behavior of body area networks at 2.4Ghz, in MOBICOM, pp. 241-252, 2009.

[9] B. Ishibashi and R. Boutaba, Topology and mobility considerations in mobile ad hoc networks, Elsevier Ah Hoc Networks, 3(6):762-776, November, 2005.

[10] E. Reusens, et al., Characterization of On-Body Communication Channel and Energy Efficient Topology Design for Wireless Body Area Networks, IEEE Transactions on Information Technology on Biomedecine, 13(6):933-945, November 2009.

[11] A. Ehyaie and M. Hashemi, and P. Khadivi, Using Relay Network to Increase Life time in Wireless Body Area Sensor Networks, IEEE WOWMOM, Kos, Greece, 15-19 June 2009.

[12] X.Liang et al., Exploiting Prediction to Enable Secure and Reliable Routing in Wireless Body Area Networks, IEEE INFOCOM, Orlando, Florida, USA, March 25-30, 2012.

[13] Q.Zhang et al., Reactive Virtual coordinate Routing Protocol for Body Sensor Networks, IEEE ICC, Ottawa, Canada, June, 2012.

[14] J.Elias and A. Mehaoua, Energy-aware Topology Design for Wireless Body Area Networks, IEEE ICC, Canada, June, 2012.

[15] R.R. Boorstyn and H. Frank, Large-scale network topological optimization, IEEE Trans. Commun., 25(1):29-47, January, 1977.

[16] H. Ren, Q.-H. M. Max, Bioeffects control in wireless biomedical sensor networks, IEEE SECON, Reston, VA, USA, September, 2006.

[17] Make Human http://www.makehuman.org/. 